\section{Isolated interrupted aortic arch complicated by subarachnoid hemorrhage in an adult patient}

\author{
Sir, \\ Interrupted aortic arch (IAA) is a rare congenital anomaly \\ characterized by complete luminal and anatomic \\ discontinuity between ascending and descending aorta. \\ Subarchnoid hemorrhage (SAH) is extremely uncommon \\ in these patients and only two children with SAH have \\ been documented earlier in the literature ${ }^{[1,2]}$ We describe \\ $\mathrm{SAH}$ in an adult patient with IAA.
}

A 32-year-old man was brought to our hospital with complaints of severe headache and vomiting of 12 hours duration. On examination, he was lethargic but able to follow commands. Both the carotids and upper limb pulses were palpable. Both femoral and popliteal pulses were weak. The blood pressure (BP) was 190/120 mmHg in the right arm and 180/110 $\mathrm{mmHg}$ in the left arm. There was also a significant difference in BP between upper and lower limbs $(120 / 80 \mathrm{mmHg})$. There was grade 3 systolic murmur on the left second intercostals area. On neurologic examination he was lethargic and was able to follow commands. There was mild nuchal rigidity. Examinations of other systems were all normal.

Transthoracic echocardiography did not show any structural cardiac anomaly except concentric left ventricular hypertrophy. Computed tomography (CT) scan of the head demonstrated SAH in the lateral fissure, cisterna ambiens, and basal cisterns. Intracranial magnetic resonance angiography (MRA) did not demonstrate any cerebral aneurysm or other anatomic etiology for the SAH.

Blood pressure was managed acutely with sodium nitroprusside. He was started on prophylactic nimodipine to prevent cerebral vasospasm and also started on epsilon-aminocaproic acid (EACA) to reduce the risk of rebleeding. On the fifteenth hospital day the patient's condition improved. Systolic BP ranged between $130 \mathrm{mmHg}$ and $160 \mathrm{mmHg}$ in the upper extremities.

Cerebral angiography was attempted but the catheter introduced through the right femoral artery could not be advanced into the aortic arch. A descending aortogram was performed that suggested a complete interruption of the aorta distal to the origin of the left subclavian artery with no washout from above [Figure 1]. CT angiography (CTA) clearly affirmed a complete interruption of the descending aorta $1.5 \mathrm{~cm}$ from the left subclavian artery with extensive collateralization such as at the prevertebral area. Additionally, internal mammarian, axillary, and intercostal arteries were dilated [Figure 2]. The patient was diagnosed as IAA type A, according to the classification of Celoria and Patton..$^{[2]}$ The diagnosis was confirmed at operation two months later. The BP came to normal after operation. He remained normotensive with no neurologic deficit during the one-year follow up.

In our patient we could not do conventional cerebral angiography due to IAA and MRA did not demonstrate any aneurysm. However, we can not completly exclude the possibility of aneurismal SAH in our patient. MRangigraphy is not the gold standard investigation for the diagnosis of cerebral aneurysm. Non-visualization of cerebral aneurysms on MRA may be relatated to several factors: Vasospasm or spontaneous thrombosis resulting in nonfilling, destruction of the aneurysms, or aneurysm sizr smaller than $6 \mathrm{~mm} \cdot{ }^{[2,3]}$

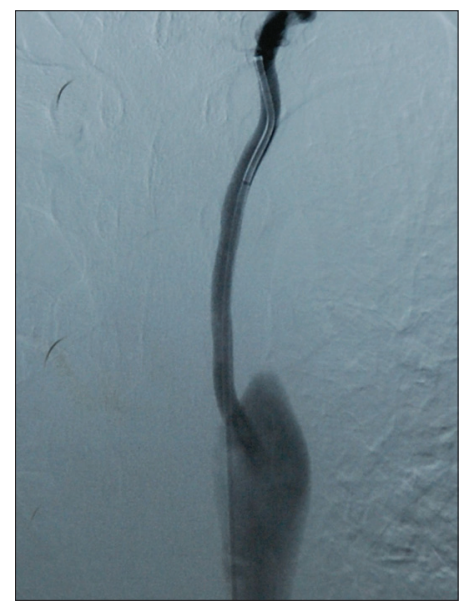

Figure 1: Descending aortogram via the left femoral artery showing complete interruption of the lumen of the descending aorta

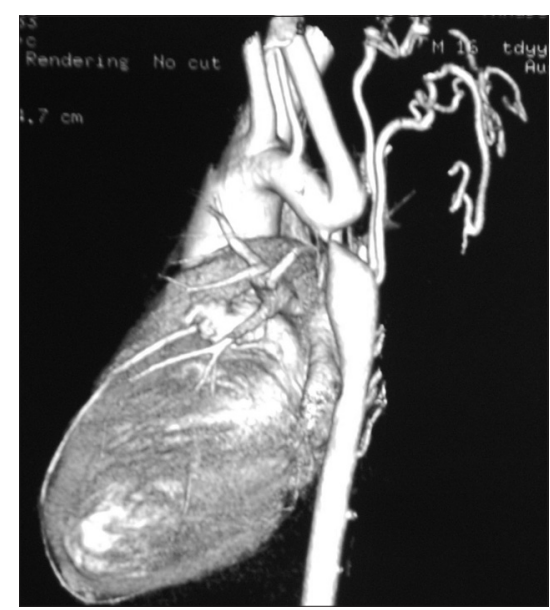

Figure 2: 3D reconstructed computed tomography angiograpy showing congenitally interrupted aortic arch with extensive collaterals 
Rebleeding is the most feared complication of SAH and EACA, an antifibrinolytic agent, is used to prevent rebleeding in aneurysmal SAH. Earlier studies using antifibrinolytic agents have clealy demonstrated the beneficial effects in reducing the risk of rebleeding. However long-term therapy was associated with increased incidence of vasospasm and no appreciable effect on mortality. More recent studies have indicated that early, short-term use of antifibrinolytic agents (less than three days) along with calcium channel blocking therapy may be beneficial. ${ }^{[4]}$ There is controversy regarding the need and magnitude of acute $\mathrm{BP}$ reduction in such patients. Reduction of BP may decrease the risk of rebleeding, however, it may result in reduced cerebral perfusion. An arbitrary cut-off of systolic blood pressure of $180 \mathrm{mmHg}$ is currently used in patients with untreated aneurysms. ${ }^{[5]}$

A complete physical examination is extremely important for the correct diagnosis of IAA. When some signs such as high blood pressure, differential BPs in the extremities, and absent or weak peripheral pulses in the lower extremities are present, isolated IAA should be suspected. Catheter angiography and CTA can be chosen to confirm the diagnosis. However, catheter angiography may be difficult to perform in patients without a prior knowledge of vascular anatomy to ensure visualization of both proximal and distal segments, as in our case. CTA with $3 \mathrm{D}$ reconstruction is a reliable noninvasive technique for the diagnosis of the aortic arch anatomy and pathology as an alternative to conventional angiography ${ }^{[6]}$

\section{Yu Hong Cao, Guang Yun Zhang', Jun Liang Han ${ }^{1}$ \\ Departments of Pediatrics and ${ }^{1}$ Neurology, Xijing Hospital, Fourth Military Medical University, Xi' an, China. E-mail: zhgyun@fmmu.edu.cn}

\section{References}

DOI: $10.4103 / 0028-3886.59485$

1. Ling F, Bao YH. Myelopathy and multiple aneurysms associated with aortic arch interruption: Case report. Neurosurgery 1994;35:310-3.

2. Sibley E, Doroshow RW, Milliken JC, Anand SK. Subarachnoid hemorrhage and isolated atresia of the aortic arch. J Emerg Med $2002 ; 22: 179-83$.

3. Vaphiades MS, Horton JA. MRA or CTA, that's the question. Surv Ophthalmol 2005;50:406-10.

4. Chwajol M, Starke RM, Kim GH, Mayer SA, Connolly ES. Antifibrinolytic therapy to prevent early rebleeding after subarachnoid hemorrhage. Neurocrit Care 2008;8:418-26.

5. Ferro JM, Canhao P, Peralta R. Update on subarachnoid haemorrhage. J Neurol 2008;255:465-79.

6. Yang DH, Goo HW, Seo DM, Yun TJ, Park JJ, Park IS, et al. Multislice CT angiography of interrupted aortic arch. Pediatr Radiol 2008;38:89-100. 